\title{
Experimental Results from Bore Phenomenon in Open Cylindrical Channel under Precession
}

\author{
Hajar Alshoufi \\ Department of Hydraulic and Water Resources Engineering, Budapest University of Technology and Economics, Budapest, Hungary \\ Email: hajar.alshoufi@emk.bme.hu
}

How to cite this paper: Alshoufi, H. (2022) Experimental Results from Bore Phenomenon in Open Cylindrical Channel under Precession. Open Journal of Fluid Dynamics, $12,69-85$

https://doi.org/10.4236/ojfd.2022.121004

Received: January 2, 2022

Accepted: March 5, 2022

Published: March 8, 2022

Copyright (c) 2022 by author(s) and Scientific Research Publishing Inc. This work is licensed under the Creative Commons Attribution International License (CC BY 4.0).

http://creativecommons.org/licenses/by/4.0/

\begin{abstract}
Experimental campaigns were carried out to extract results from the flow in an open cylindrical channel under precession. The bore or the hydraulic jump is the main concentration. The experimental results are varied; this includes velocity results and geometrical ones related to the depths, phases, and lengths. For the geometrical ones a Coupled-Charged Device Camera (CCD) is used to extract pictures, those enable us to get quantitative and qualitative results. For the velocity results, Acoustic Doppler Velocimeter (ADV) is used to extract the velocity signals under the bore surface, after analyzing them it turned out that they have Cnoidal form, thus a new BBM model is derived, which is exactly as the one derived by Peregrine (1966), the only difference is the forcing gravity term, this model is solved analytically after omitting this gravity term as it is considered small, and the solution is compared with the real signals with good match. Finally, a new relationship that connects between the conjugate depths after and before the bore is derived which has time-space dependency due to the Centrifugal effect, it was also used and compared with some experimental results.
\end{abstract}

\section{Keywords}

Bore, Precession, CCD Camera, ADV Velocimeter, Cnoidal Waves

\section{Introduction}

It is well known that the transition between two levels of water have different aspects, it can be totally broken highly agitated and turbulent which is wellknown phenomenon as bore or hydraulic jump, and sometimes this level is covered by smooth periodic undulations called the dispersive shock waves or undular bores [1]. In the first case, the energy is totally dissipated by friction, while in the second one part of this energy is radiated in an undular form [2]. The main 
experimental achievements on bore problem are varied, the earliest experiments were conducted by Favre (1935) who studied the surge wave propagating from the reservoir, his results were summarized in [3], the authors stated that after specific time some undulations take place on the free surface, those have stable form and similar in size and of Cnoidal type of waves.

The experiments of Favre stimulated other researchers to study this topic, for instance, in [4] the authors performed two experimental campaigns on the surges moving from a reservoir through rectangular contraction into an open channel that was given different slopes, the first one used a light metal planing plate with different loads over the surface to maintain the first wave, and then a train of undular waves can appear. And in the second one, they used an external weir, when the weir was slowly raised into a stream for which the Froude number exceeded unity, the water being deflected into a stable hump above the weir. The authors stated that above Froude number of 1.3 the first wave is broken locally, its type is determined using Ursell number, they also compared the results with the classical momentum conservation theory. Other experiments were carried out in [5], the main concentration was the surface configuration of breakers, bores, and hydraulic jumps in flumes with transparant sides, which are considered different modes of quasi-steady breaking waves, their observations indicates that the surface roller does not play a dominant role in the dynamics of the wave.

The bore can be found naturally in rivers, intensive field investigations of tidal bore dynamics conducted during several months in the Garonne River (France) in [6], it turned out that tidal bores form for a large majority of tides, with an occurrence percentage of $90 \%$ for low flow dishcarge and $65 \%$ for large flow discharge, their results shed the light also on the significant cross-section variability of undular bores that contrasts with rectangular channel experiments, which in turn pours in favor the experiments conducted in [7] for the trapezoidal channels. In [7] the author investigated the undular bores (Favre's waves as he called them) in detailed measurements for both rectangular and trapezoidal flumes, the author tracked the different type of waves observed in both channels depening on the value of Froude number that he extracted from the conservation of momentum and continuity. Along the channel, many gauges were inserted in different places, which enabled him to draw the hydrographs for both the positive and negative surges respectively. The new facilities that are used in studying bore experimentally are varied, for instance in [8] the author used the LIF (Laser-induced fluorescence) and PIV particle image velocimetry to extract velocity and depth results, their main concentration is to study the hydrodynamics of largescale bore driven swash on impermeable rough beach slopes. Those facilities enabled the authors, for instance, to extract depth time-series, depth-averaged velocities, Reynolds stress profilers, depth-averaged turbulent kinetic energy etc. Their measurements resolve the backwash shoreline position and the late backwash period, when depths are shallow and velocities are high.

Other type of bore in reality is the Tsunami bore, in [9] the authors carried 
out extensive experiments using the dam-break waves to simulate the interaction between the tsunami-like bore and vertical seawall to measure the bore-induced pressures and to estimate forces exerted on the vertical seawall model, where the force distribution is affected by the front impact phase, and quasi-steady one, in addition to bore run-up, and overflow phase. Other experimental and numerical results extracted on dam-breaking bores are examined in [10] the author's model was originally used to simulate debris-flow collision on plane walls. His experiments showed a lag time between the maximum wall force and the maximum run-up elevation. The author used numerical analysis as well where Finite Volume Element scheme is implemented to the potential flow problem, the model is compared with the experimental measurements. The experiments of Treske stimulated many researchers to carry out intensive experimental and analytical investigations on undular bore development, like for instance in channels of variable cross-section conducted in [11], the authors discussed mainly two cases: the high Froude number (HFN) regime where in the channel axis short waves with characteristics similar to those Favre's waves were observed, and in the channel banks longer waves were observed, and the low Froude number (LFN) regime where only the long waves were observed in the whole channel. The experiments focused on comparing their numerical model of Serre-Green-Naghdi model with other work related to the wavelength, amplitudes, and steepness with good match.

The real observations in rivers showed that the turbulent mixing and dispersion are enhanced by the bore because some significant bed erosion and scour take place beneath the bore while suspended material are advected upstream with the tidal bore [12]. That is why in [13] the authors carried out extensive experimental measurements to study the turbulent velocity in breaking bores and the turbulence properties, they discussed the free surface properties based on Froude number and the velocity which was extracted using Acoustic Doppler Velocimeter (ADV) which showed that some negative longitudinal velocity components next to the bed highlighting some transient recirculation „bubble”. It was noticed that there is an increment in Reynolds stress between the uniform area behind the bore to the bore area, for both smooth bed material or the fixed gravel bed.

In the present work and due to the precession mechanism that provides sudden change and push to the free surface elevation, the bore is natural, sometimes with totally turbulent broken area other times with smooth undulations. In this paper, we shed the light on some experimental results extracted from the bore noticed in open cylindrical channel under precession. First by tracking the different free surface aspects and flow patterns based on the classical classifications, and next by extracting a new BBM model similar to the one extracted in [14] which is going to be solved analytically to compare it with the results extracted from the ADV measurements for the undular breaking and breaking bore cases that were carried out in the channel and it showed the cnoidal feature of the ve- 
locity along the flume. In Section 2, explanation about the experimental setup, in Section 3, explanation about the experimental observations for the free surface and the velocity measurement, in Section 4, the new BBM model is derived and compared with the experiment, in Section 5, the conclusion and summarization of the work.

\section{Experimental Setup}

A flume channel of cylindrical geometry that has an annulus cross-section is elevated over the laboratory floor (the inertial frame of reference) using metal support, is designed to study the forced oscillations. The cylinder width $r$ is the difference between the radii of the inner and the outer cylinders $r_{\min }, r_{\max }$, respectively. Full details of the experimental apparatus and the procedure can be found in [15]. The base of the cylinders is a rounded-shaped wood table that rotates and tilts, which is in turn elevated over other rotating circular wood table that can only rotate. The cylinders rotate about their common axis $(O, \hat{k})$ with precession rate $\Omega_{2}=\tau \Omega$, where $\tau$ the slope of the upper base table. The lower table is able to rotate about the inertial axis $(O, z)$ with rotation rate $\Omega$. The angle between the local axis $\hat{k}$ and the inertial one $z$ is the angle of precession $\psi$. The cylinders are filled with water of different volumes, Figure 1 shows the channel.

\section{Experimental Observations}

The experimental observations are divided into two parts, first for the free surface, and then for the velocity measurements and observations.
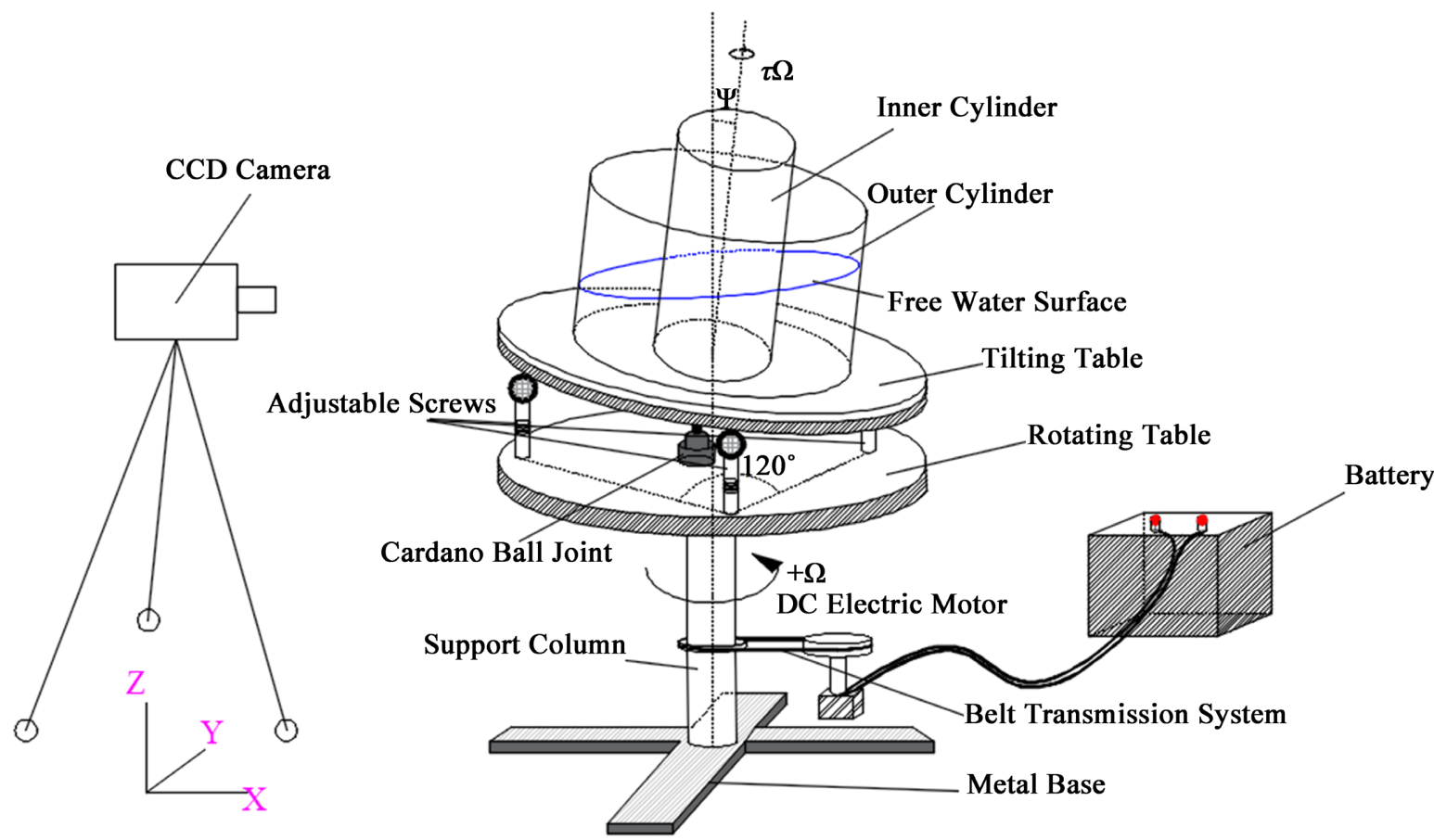

Figure 1. Sketch of the channel flume used in the experiments to track the wave aspects observed. 


\subsection{Free Surface Observations}

We start by the free surface observations, where this is considered the first experiment on the bore in such channel system as far as we know. But first we shed the light on the techniques we used to extract the quantitative and qualitative results. In order to measure any length for instance the depths on the outer periphery of the flume, we used the pictures extracted from the CCD camera that was fixed firmly on the laboratory floor in front of the channel to record continuous pictures of what happen inside the flume. The pictures will give us the pixels on the pictures. In order to get the right $\mathrm{mm}$ depths and lengths, we needed to know the corrected pixels and the right $\mathrm{mm}$.

\section{1) Millimetres Computations:}

To get the real $\mathrm{mm}$ results on the outer periphery, we used a technique depends on pasting some small square papers, those were given specific numbers, and they were organized in a grid form on the outer surface this grid has some origin, the chosen one was point number 5 , the distances were measured between the points and the origin and depending on some initial guess for their positions with respect to this point, we could minimize the distance relationship $d=\sqrt{\left(x-x_{c}\right)^{2}+\left(y-y_{c}\right)^{2}}$ using the least square method where we minimized the error between the real distances and the computed ones based on new computed ordinates and abscissas iteratively, so that we got the exact ordinates and abscissas for each point.

\section{2) Pixels Computations:}

To extract the right pixels on the pictures, first they were calibrated, where new program is written by the present author using Python programming language to calibrate them, then using the thresholding technique provided by Python packages, we could get the Gray-Scale image, and then by using the bounding techniques we could detect the centres of the squares pasted on the outer periphery (x-pixel and y-pixel) which have known $\mathrm{mm}$ from the previous section. Now in order to compute a length or distance, we use the curve tracking techniques and then we extract again the pixels needed and then using the interpolation and extrapolation techniques between the pixels and the real $\mathrm{mm}$ we could draw the free surface in $\mathrm{mm}$ or get the right length as it appears on the outer periphery. From the curve tracking techniques, we could draw the free surface when the undular bore case as it is clear in Figure 2 which reflects the cnoidal type of the waves.

The cases of the bore free surface are similar to the ones noticed under nonrotating conditions, which means that one can find the totally broken bore, the undular one and the undular bore with first wave is broken. Those cases were classified previously in [4] [14] [16], the main criterion was the level difference between the two uniform flows after and behind the bore, that is given:

$$
\Delta=\frac{h_{1}-h_{2}}{h_{2}}
$$


Here $h_{1}, h_{2}$ represents the uniform depths before and after the bore. It is agreed that if this level is $\Delta>0.75$ then the connected region between the two uniform flows is totally broken (bore) or plain bore (appears as slanted roller), on the other hand if $0.3<\Delta<0.75$ then the free surface is undular, but the first wave is broken, and for smaller ratio like $\Delta<0.3$ the free surface is totally smooth undular. In the present work, the channel is cylindrical one and tilted, the uniform area behind and after the bore were only noticed for the cases of small tilt, for bigger angles of tilt like $\tau=0.09667$, and strong rotation rate the free surface behind the bore was totally curved towards the inner cylinder of the flume with highly agitated area as it is clear in Figure 3.

And in fact, we cannot say that we have really totally plain area, there should

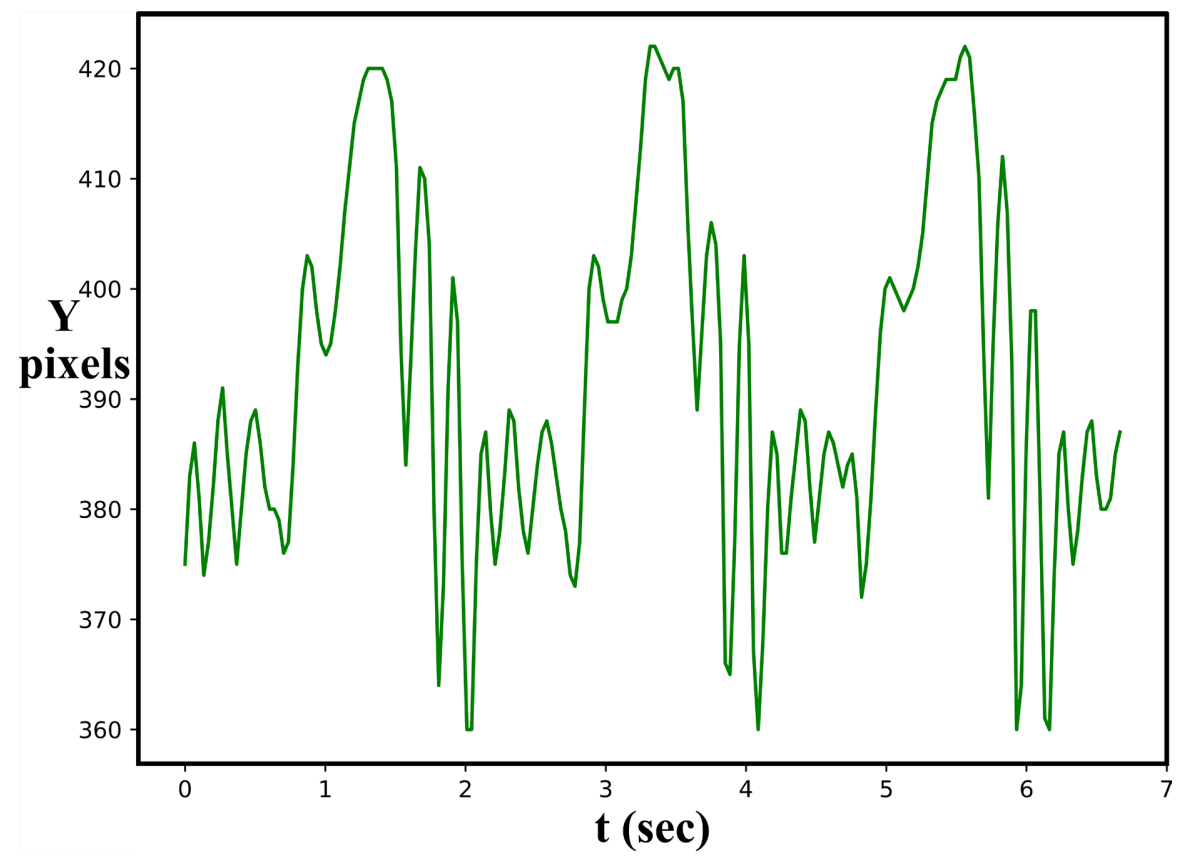

Figure 2. Free Surface observed at specific point on the flow free surface for undular bore case with time, $V=6000 \mathrm{ml}, \tau=0.0433, \Omega=3 \mathrm{rad} / \mathrm{s}$.

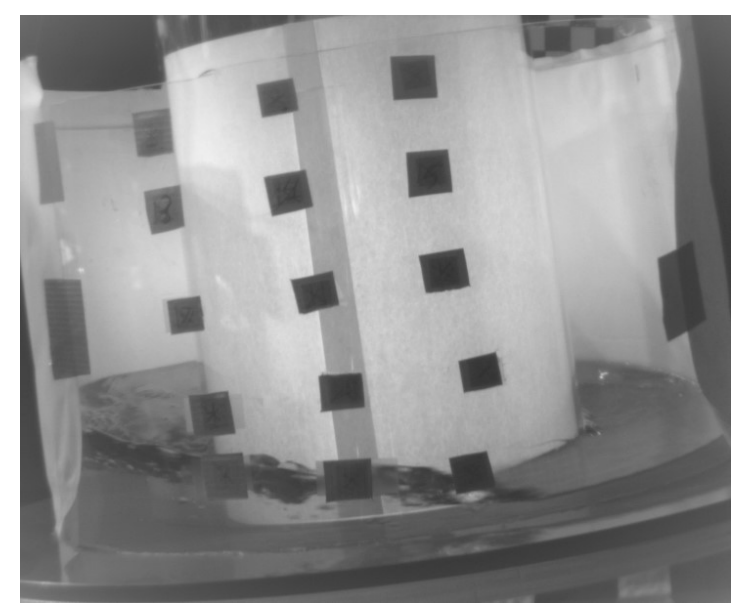

Figure 3. Breaking bore, $V=4000 \mathrm{ml}, \tau=0.09667, \Omega=4.893 \mathrm{rad} / \mathrm{s}$. 
be small ripples appear on the surface especially that the motion is sinusoidal one in the whole flume. But the classification in Equation (1) was tested for many cases observed in the flume. To test the validity of Equation (1) more than 50 samples were taken, using the calibrated pictures and the computation process we followed above, we could determine the main properties of the jump like $h_{1}$, $h_{2}, L_{j}$, the phase, the height of the jump etc. we have to explain first about $h_{1}$ where the free surface behind the bore appears as if it is slightly narrowing to the bore area, thus we cannot say that it is exactly uniform that is why we take three points behind the bore and then we take their averaged depth to get the right depth, on the other hand the case of $h_{2}$ appears from the figures as if the depth is uniform and no need to take any averaging. It is worth to mention that for the case of undular bore $h_{1}$ (the bigger depth) was computed from the average depth of water which satisfies the average water level constraint:

$$
V=\int_{0}^{2 \pi} \int_{r_{\min }}^{r_{\max }} \int_{0}^{\bar{h}=H+\eta(t, \theta, r)} r \mathrm{~d} z \mathrm{~d} r \mathrm{~d} \theta=\bar{h}\left(r_{\max }^{2}-r_{\min }^{2}\right) \pi
$$

where $r, \theta, z$ the radial, azimuthal and axial cylindrical coordinates, respectively. The results from Equation (1) were in general in good agreement with the normal classifications (under nonrotating conditions), however only the undular cases were divergent, for instance the case of totally broken bore or the plain bore in Figure 4, has level ratio $\Delta=1.09>0.75$, on the other hand as clear in Figure 5 the bore looks undular but locally broken at the first wave, and this case satisfies the ratio $0.3<\Delta=0.641<0.75$. Finally, in the undular case, it was observed by the naked eye that there is small stair of waves, but the level difference looks too small as clear in Figure 6 however the classical approach is broken where the ratio $\Delta=0.525>0.28$. In all cases, the base of the upper titling table is assumed the origin of the vertical depth.

Most of the previous studies connected the breaking property of the undular bore with Froude number, for instance in [13] they found that the bore was broken if $F_{r}>1.5-1.6$, and in [4] they got the first breaking wave at about $F_{r} \approx 1.3$. Thus, each experiment is different and gives different results, in the

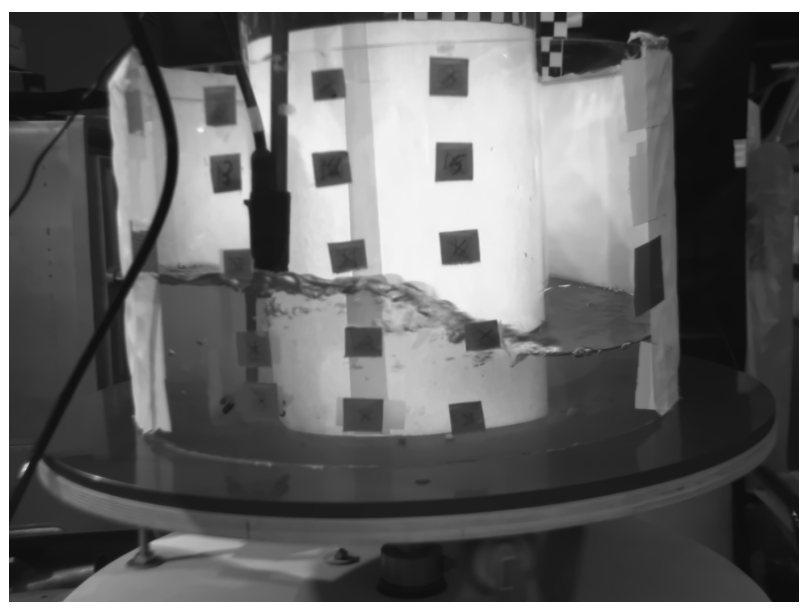

Figure 4. Plain bore, $V=10000 \mathrm{ml}, \tau=0.04333, \Omega=4.71 \mathrm{rad} / \mathrm{s}$. 


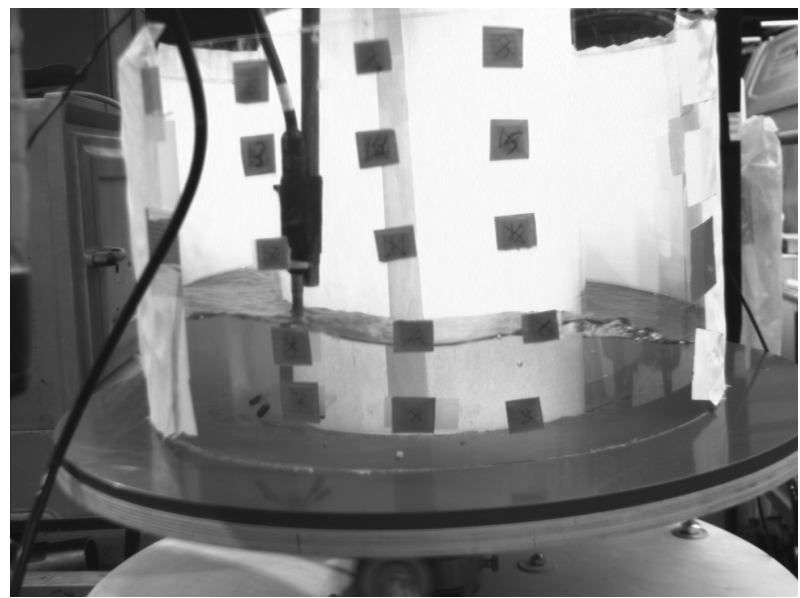

Figure 5. Undular broken bore, $V=8000 \mathrm{ml}, \tau=0.04333, \Omega=3.824 \mathrm{rad} / \mathrm{s}$.

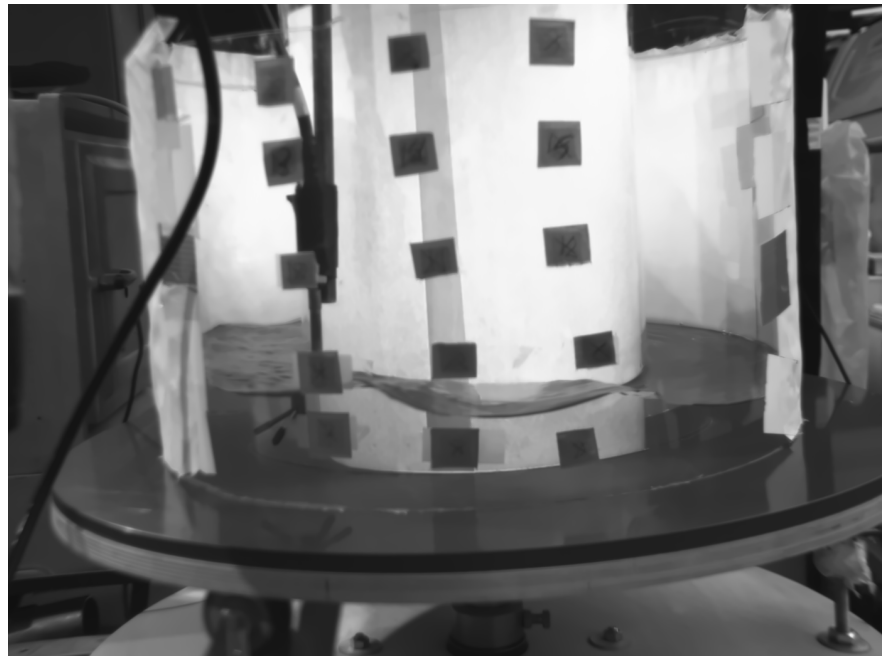

Figure 6. Undular bore, $V=6000 \mathrm{ml}, \tau=0.04333, \Omega=3 \mathrm{rad} / \mathrm{s}$.

present study the flow in the whole flume is supercritical downstream and critical upstream with some subcritical cases almost in all cases, because Froude number is given in this case in terms of the solid-body rotation speed as in Equation (3), Some results were reported in Table 1 about the tilt, rotation rate, volume, and the corresponding value of Froude number.

$$
F_{r}=\frac{\Omega r_{\max }}{\sqrt{g h_{2}}}
$$

The observer of the transverse direction of the flow (the radial one) will see in case of slow rotation a jump of the form similar to the one under nonrotating conditions, however by increasing the rotation rate the frontal line of the bore is inclined inward towards the inner radius of the channel as it is clear in Figure 7, this result is similar to the one extracted in [17] for his measurements on the rotation effect on the hydraulic jump in rectangular channel that is elevated on a rotating table, the author justified this deflection to the right to the Coriolis acceleration effect, this is also the result was reached in [18] but their experiment 
Table 1. Experiment studies of unsteady bore.

\begin{tabular}{ccccccc}
\hline$V(\mathrm{ml})$ & $H$ & $\boldsymbol{\tau}$ & $\boldsymbol{\Omega}$ & case & $F_{\boldsymbol{T}}$ & $\Delta$ \\
\hline 10,000 & 0.0933 & 0.0433 & 5.092 & plain bore & 1.5 & 1.108 \\
8000 & 0.074 & 0.0433 & 3.842 & undular broken & 1.29 & 0.675 \\
12,000 & 0.112 & 0.0433 & 5.045 & plain bore & 1.32 & 0.882 \\
6000 & 0.056 & 0.0583 & 2.884 & undular bore & 1.19 & 0.874 \\
4000 & 0.037 & 0.0333 & 4.404 & plain bore & 2.22 & 1.14 \\
14,000 & 0.13 & 0.075 & 5.66 & undular broken & 1.5 & 1.03 \\
2000 & 0.0185 & 0.0333 & 3.88 & plain bore & 2.22 & 1.25
\end{tabular}

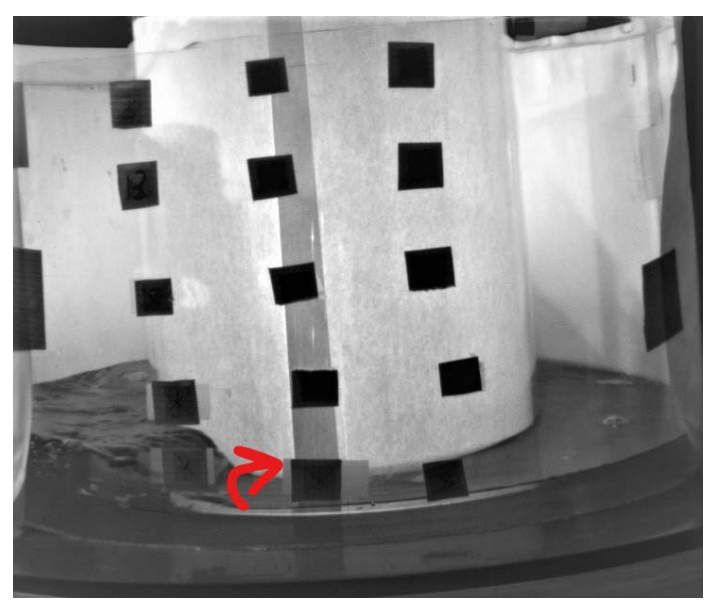

(a)

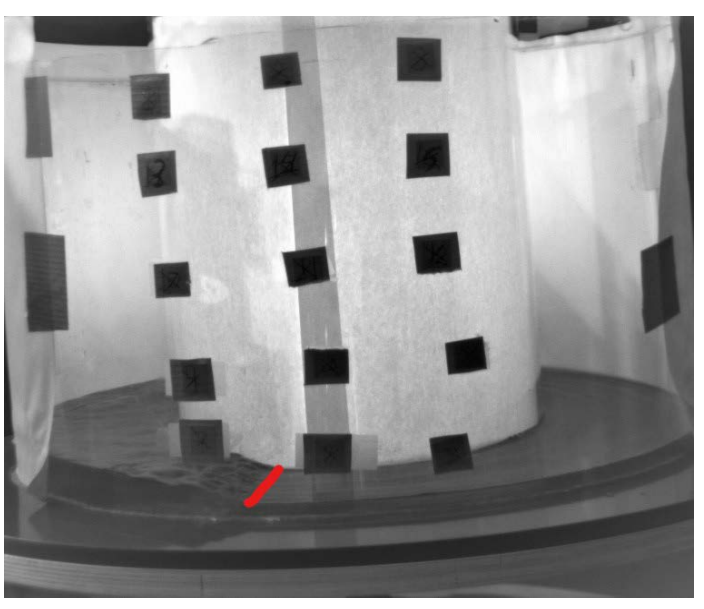

(b)

Figure 7. (a) Plan view of propagating hydraulic jump to the right due to high rotatio rate, $V=4000 \mathrm{ml}$, $\tau=0.0333, \Omega=4.403 \mathrm{rad} / \mathrm{s}$. (b) Plan view of propagating hydraulic jump straight under low rotation rate, $V=2000 \mathrm{ml}, \tau=0.0333, \Omega=2.785 \mathrm{rad} / \mathrm{s}$.

was little different than the result extracted in [17], where they studied the jump in a semi-infinite ocean, and they could determine the exact angle of that oblique offshore which depends on the strength of the jump, this results contradicts the one observed in [17] where the angle mainly depends on the rotation speed, the work here accords with Fedorov's results where the effect of rotation rate plays the role in this deflection with the other factors like the tilt and the volume, the analytical calculations of this deflection was not considered in this work.

\subsection{Conjugate Depths}

Water levels before and after the breaking area are called the conjugate depths; in normal channels there is a relationship between those depths, which can be written either in terms of Froude number or the volume flow rate per unit width. This relationship is going to be derived here as well by taking into account the additional forces resulting from rotation; by applying momentum conservation the problem can be written as:

$$
\sum \vec{F}=\rho Q\left(\vec{V}_{2}-\vec{V}_{1}\right)
$$


Here, $V_{1}$ denotes the velocity of the uniform flow propagating at higher level $h_{1}$. $V_{2}$ represents the velocity of the uniform stream after the bore at level $h_{2}$. The velocity at section (1) in the channel corresponds to the case of subcritical conditions, and to the supercritical conditions at section (2). As in any simple channel, the forces that affect the flow mainly the pressure and gravity, but other forces resulting from rotation should be added as well. Euler wobbling force, for instance, is small in comparison with the Centrifugal one; thus, it is neglected. The motion takes place in one direction of the flow, the azimuthal one. In this azimuthal projection, there is Coriolis force term that has vertical velocity component, but due to the small width this velocity can be neglected, that is why this projection is factored out, the rest is only the Centrifugal force and one can simply write:

$$
\begin{aligned}
& \gamma \bar{h}_{1} A_{1}-\gamma \bar{h}_{2} A_{2}+\gamma \tau r \sin (\Omega t-\theta)\left(\frac{h_{1}+h_{2}}{2}\right) L_{j} \\
& -\left[\gamma r \Omega^{2} \tau^{2} \sin (\Omega t-\theta) \cos (\Omega t-\theta)\right]\left(\frac{h_{1}+h_{2}}{2}\right) L_{j}=\frac{Q^{2}}{g r}\left(\frac{h_{1}-h_{2}}{h_{1} h_{2}}\right)
\end{aligned}
$$

The cross-section of this channel is a rectangular one that has width $r=r_{\max }-r_{\min } \cdot \bar{h}_{1}, \bar{h}_{2}$ the distances from the centroids of water sections (before and after the jump) till the free surface of water are given by:

$$
\bar{h}_{1}=\frac{h_{1}}{2} \cos (\psi), \bar{h}_{2}=\frac{h_{1}}{2} \cos (\psi)
$$

where $\psi$ the angle of tilt in the channel. $L_{j}$ the jump length. $A_{1}, A_{2}$ the water section areas before and after the jump, and are given by:

$$
A_{1}=r \cdot h_{1}, A_{2}=r \cdot h_{2}
$$

$\Omega$ the angular velocity, the rotation rate about the $z$ axis, $\rho$ the density of water in the channel. The Froude number in this channel can be given for the section before the jump:

$$
F_{r 1}^{2}=\frac{Q^{2} r}{g \cdot A_{1}^{3}}
$$

By arranging Equation (5) in terms of the proposed depths we get a simple quadratic equation:

$$
\begin{gathered}
\left(\frac{h_{2}}{h_{1}}\right)^{2}+\left(\frac{h_{2}}{h_{1}}\right)-2 G_{2}^{2}=0 \\
G_{2}^{2}=\frac{F_{r 1}^{2}}{G-G_{1}}, G=\cos (\psi)+\frac{\tau L_{j} \sin (\Omega t-\theta)}{h_{1}-h_{2}} \\
G_{1}=\frac{\Omega^{2} \tau^{2} L_{j}}{h_{1}-h_{2}} \sin (\Omega t-\theta) \cos (\Omega t-\theta)
\end{gathered}
$$

Thus, to find the conjugate depths the determinant of Equation (9) will give two different values.

$$
\frac{d_{2}}{d_{1}}=\frac{1}{2}\left[-1+\sqrt{1+8 G_{2}^{2}}\right]
$$


If the channel is totally horizontal $\psi=0$, then $\tau=0, G_{2}=\frac{F_{r 1}^{2}}{\cos (\psi)}$ and Equation (12) will be similar to the cases of conjugate depths under normal conditions:

$$
\frac{d_{2}}{d_{1}}=\frac{1}{2}\left[\sqrt{1+8 F_{r}^{2}}-1\right]
$$

Equation (12) is going to give each time different result depending on the phase of tilt $\Omega t$ and the azimuthal coordinates $\theta$, for instance the computations here first determined the length of the jump on the outer surface from which we could compute the angle $\theta$ in terms of $r_{\max }$, this corresponds to specific time which accords with the figure extracted, as the CCD camera is taking continuous figures at specific rate 30 frames per second and each frame has specific number, by counting the number of frames that are recordered from the starting time till the wanted picture we can calculate the time. By choosing some arbitrary cases, we put this relation in Figure 8 compared with the classical momentum in Equation (23) and the experiments.

\subsection{Velocity Observations}

The velocity was recorded as well while bore is on the free surface. The experiment utility was the ADV or the Acoustic Doppler Velocimeter, the device is composed of four heads surrounded the transmit transducer, and it was hung on a metal rod next to the channel where it can be hold and fixed and it is inserted

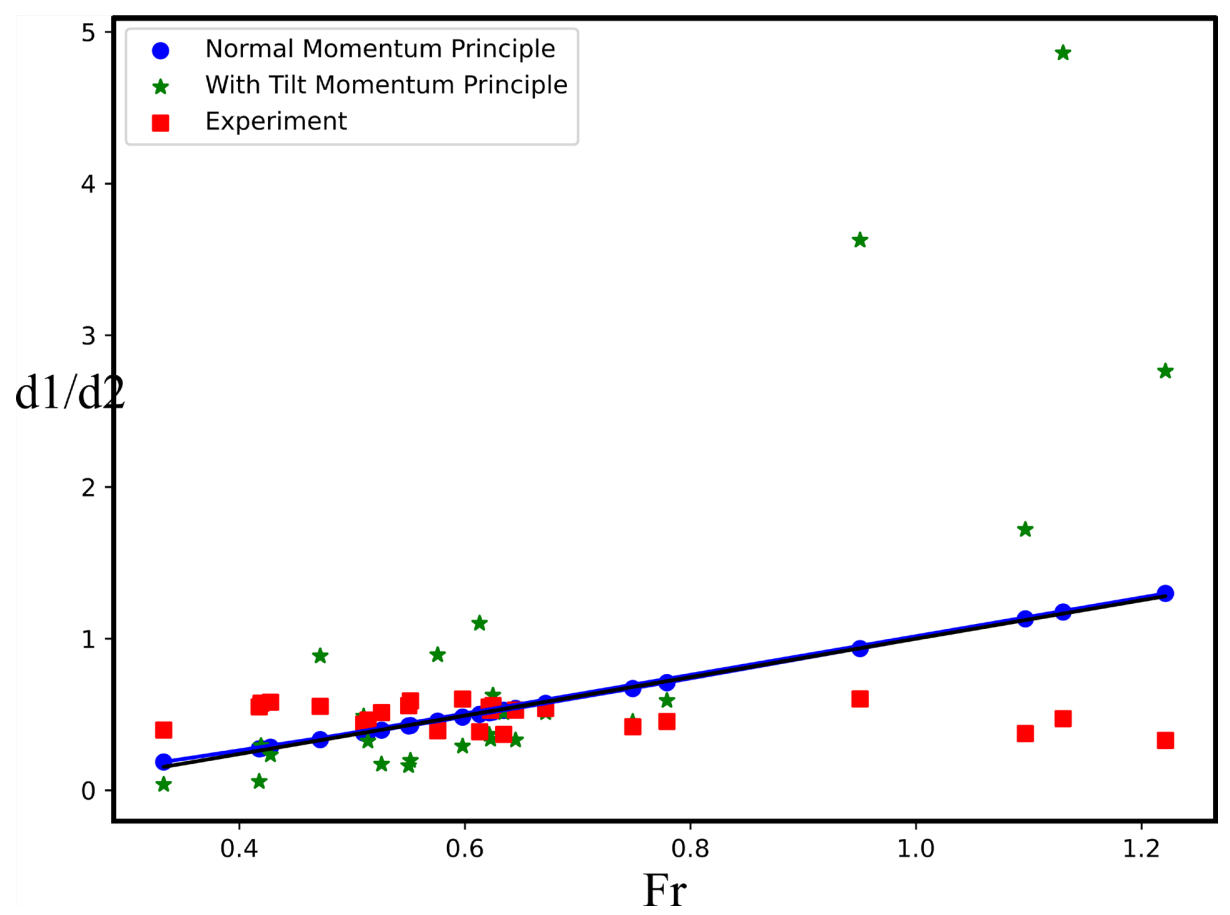

Figure 8. Ratio of conjugate depths as a function of the bore Froude number. $\left(^{*}\right)$ using Equation (12), ( $\bullet$ experiments, (•) using Equation (13). The solid line is linear fit for the momentum principle. 
in the channel. The channel width is only $10 \mathrm{~cm}$, and the channel was tilted using different angles of tilt, for big ones and during the rotational motion the rod that holds the ADV scratched the inner cylinder, thus it was hung all the time almost in the centre line of the flume $r_{0}=0.045 \mathrm{~m}$. The position of the vectrino vertically also should be taken into account, where making it next to the free surface during the motion can be out of water and the signal will be full of spikes and bad results, this also may happen by making it close to the bed where the reflected signal will be very bad by the effect of bottom conditions, that is why it is advised to keep it far from the bottom about $3 \mathrm{~cm}$ and from the free surface about $2-3 \mathrm{~cm}$ to never touch the air. To enhance the correlation level (over 70\%), some seeding materials were used, mainly sand of diameter $d=0.25 \mathrm{~mm}$, the sampling frequency was $25 \mathrm{~Hz}$. The vectrino does not move or tilt with the flume but it is affected by the motion of the flow over it, the extracted results had spikes that should be corrected using averaging or interpolation and extrapolation techniques. The signals were for long time like 100 s or more, thus, to figure out their type those were divided into smaller parts like only 5 - $10 \mathrm{~s}$. To analyse the signals, we used ADV Converter and MATLAB reader to extract the velocities in numbers, the signals are all of Cnoidal form when splitting them into small time intervals.

\section{Benjamin-Bona-Mahony (BBM) Model}

As mentioned in the previous section that the bore signals are cnoidal ones, thus, in this section a new BBM model similar to the one extracted in [14] is derived and compared with many cases of tilts and different volumes. To handle this, the motion is assumed to be two-dimensional, and the predominant direction of the flow is the azimuthal one, the flow is assumed inviscid one, only Coriolis force is taken from the rotational forces, and Navier-Stokes equations with the kinematic and continuity conditions are:

$$
\begin{gathered}
u_{t}+\frac{u u_{\theta}}{r}+w u_{z}=g \tau \sin (\Omega t-\theta)-\frac{P_{\theta}}{\rho r}-2 \Omega \tau \cos (\Omega t-\theta) w \\
w_{t}+\frac{u w_{\theta}}{r}+w w_{z}=-g-\frac{P_{z}}{\rho}+2 \Omega \tau \cos (\Omega t-\theta) u \\
w=\eta_{t}+\frac{u \eta_{\theta}}{r}, u_{\theta}+r w_{z}=0
\end{gathered}
$$

where $u(\theta, z, t), w(\theta, z, t)$ the velocity components in the azimuthal and axial directions, respectively, $P$ the pressure, $g$ the gravity acceleration, $r$ radial coordinate. The dimensionless analysis is assumed:

$$
\begin{aligned}
& r \rightarrow \frac{H}{\delta} \bar{r}, \eta \rightarrow \varepsilon H \bar{\eta}, \tau \rightarrow \varepsilon \delta \bar{\tau}, \theta \rightarrow \bar{\theta}, z \rightarrow H \bar{z}, \\
& P \rightarrow P_{0}+\gamma(H-z)+\varepsilon \rho g H \bar{P}, \Omega \rightarrow \frac{c \delta}{H} \bar{\Omega}, t \rightarrow \frac{H}{\delta c} \bar{t}, \\
& w \rightarrow \varepsilon^{2} \frac{c}{\delta} \bar{w}, u \rightarrow \varepsilon c \bar{u}
\end{aligned}
$$


With: $\delta=\frac{H}{\lambda}$ the shallowness parameter, and $c=\sqrt{g H}$ the long wave speed, the wavelength $\lambda=2 \pi r_{\max }$. The equations will take the final form after dropping the bars and balancing the shallowness with the amplitude parameters in the form $\varepsilon=O\left(\delta^{2}\right)$ :

$$
\begin{gathered}
u_{\theta}+r w_{z}=0 \\
w=\eta_{t}+\varepsilon \frac{u \eta_{\theta}}{r} \\
u_{t}+\varepsilon\left[\frac{u u_{\theta}}{r}+w u_{z}\right]=\tau \sin (\Omega t-\theta)-\frac{P_{\theta}}{r}+\varepsilon^{2}[-2 \Omega \tau \cos (\Omega t-\theta) w] \\
\varepsilon\left[w_{t}+\varepsilon\left(\frac{u w_{\theta}}{r}+w w_{z}\right)\right]=-P_{z}+\varepsilon^{2}[2 \Omega \tau \cos (\Omega t-\theta) u]
\end{gathered}
$$

The unknown parameters $B(\theta, z, t)=(P, w, u, \eta)$ in Equations (18)-(21) are going to be expanded in a series form in terms of the amplitude parameter:

$$
B=B^{(0)}+\varepsilon B^{(1)}+\varepsilon^{2} B^{(2)}+\cdots
$$

At the leading order of the problem, it is assumed that as $\varepsilon \rightarrow 0$, then $\tau \rightarrow 0$, so that we get rid of the gravity term at the initial state, then for the next order we have:

$$
\begin{gathered}
u_{1 \theta}+r w_{1 z}=0, \quad w_{1}+\eta_{0} w_{0 z}=\eta_{1 t}+\frac{u_{0} \eta_{0 \theta}}{r} \\
u_{1 t}+\frac{u_{0} u_{0 \theta}}{r}=\tau \sin (\Omega t-\theta)-\frac{P_{1 \theta}}{r} \\
P_{1}=\left(\frac{z^{2}-1}{2 r}\right) u_{0 \theta t}+\eta_{1}
\end{gathered}
$$

On substituting the values of the zeroth order of azimuthal velocity into Equation (24) and assuming that: $u=\eta$, and then by adding it to the balanced version between continuity and the kinematic condition we get the final BBM model of the problem which is the same as in [14] but with forcing term from gravity force in the cylindrical geometry:

$$
u_{t}+\frac{3 u u_{\theta}}{2 r}+\frac{u_{\theta}}{r}=\frac{\tau}{2} \sin (\Omega t-\theta)+\frac{u_{\theta \theta t}}{6 r^{2}}
$$

This equation should be scaled back into the dimensional form to get:

$$
A \cdot u_{t}+B \cdot u u_{\theta}+C \cdot u_{\theta}-D \cdot u_{\theta \theta t}=E
$$

The forcing term will be neglected in the process of the direct integration due to the small values of the slope in all cases. The coefficients $A, B, C, D$ are given by:

$$
\left\{\begin{array}{l}
A=\frac{H}{\varepsilon \delta c^{2}}, B=\frac{3 H}{2 c^{2} \varepsilon^{2} \delta r}, C=\frac{H}{c \varepsilon \delta r} \\
D=\frac{H^{3}}{6 \varepsilon \delta^{3} c^{2} r^{2}}, E=\frac{\tau}{2 \varepsilon \delta} \sin (\Omega t-\theta)
\end{array}\right.
$$




\section{Analytical Solution}

The classical direct integration of Equation (27) can be processed by assuming that we have a progressing solution in a frame moving with the wave on path $\vartheta$ so that $u=u(\vartheta)$, and $\vartheta=r \theta-r \Omega t$. This means that:

$$
\frac{\partial}{\partial \theta}=r \frac{\partial}{\partial \vartheta}, \frac{\partial}{\partial t}=-\Omega r \frac{\partial}{\partial \vartheta}
$$

Then Equation (27) can be written as:

$$
(C-\Omega A) u_{\vartheta}+B u u_{\vartheta}+\Omega D r^{2} u_{\vartheta \vartheta \vartheta}=0
$$

By following the classical integration method like the one presented by Debnath (2012) the final solution can be written in the cniodal form as:

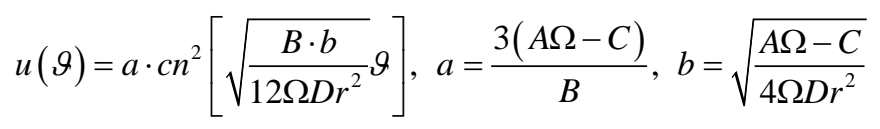

The solution in Equation (31) can be approximated into:

$$
u(\theta, t)=\beta a[1 \pm \cos (k r \theta-\omega t)]^{2}
$$

where $\beta$ is ratio to be determined from the experiment $0<\beta \leq 1$, in general it is $0.5, k$ and $\omega$ are given by:

$$
k=\sqrt{\frac{B \cdot b}{3 \Omega D r^{2}}}, \omega=\frac{C}{A}+\frac{B(2 a-b)}{3 A}
$$

The signals extracted from the previous section will be compared with the solution in Equation (32) where most of the cases reported below are of the type undular breaking or totally breaking bore cases. The solutions in Figures 9-11 with satisfactory match:

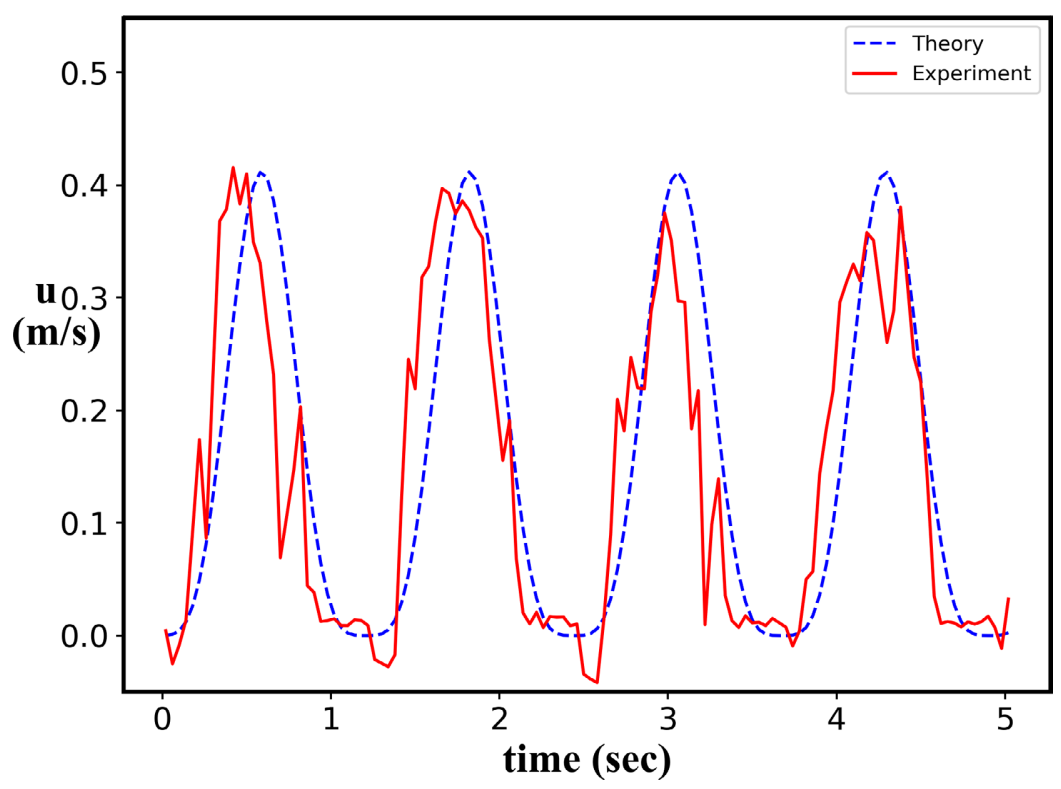

Figure 9. Cnoidal Wave solution for type of wave behind the bore, $\bar{h}=0.112 \mathrm{~m}$, $\Omega=5.05 \mathrm{rad} / \mathrm{s}, \omega=5.15 \mathrm{rad} / \mathrm{s}, \tau=0.0433$. 


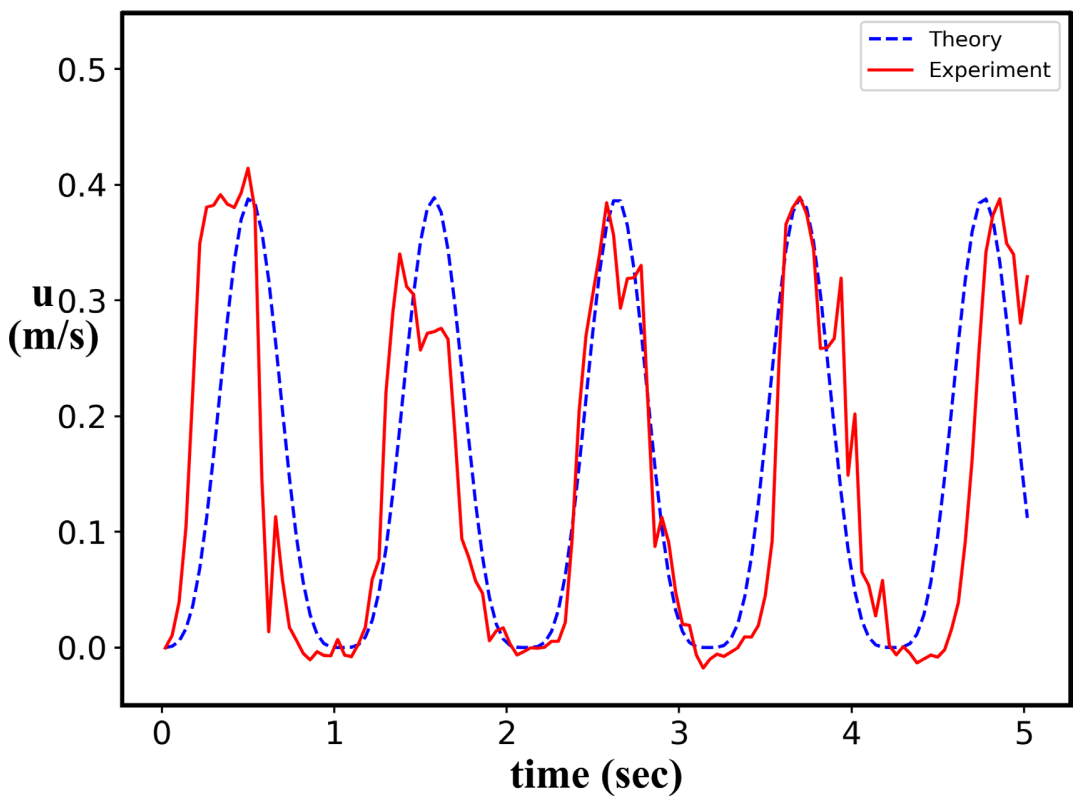

Figure 10. Cnoidal Wave solution for type of wave behind the bore, $\bar{h}=0.0933 \mathrm{~m}$, $\Omega=5.31 \mathrm{rad} / \mathrm{s}, \omega=5.952 \mathrm{rad} / \mathrm{s}, \quad \tau=0.0233$.

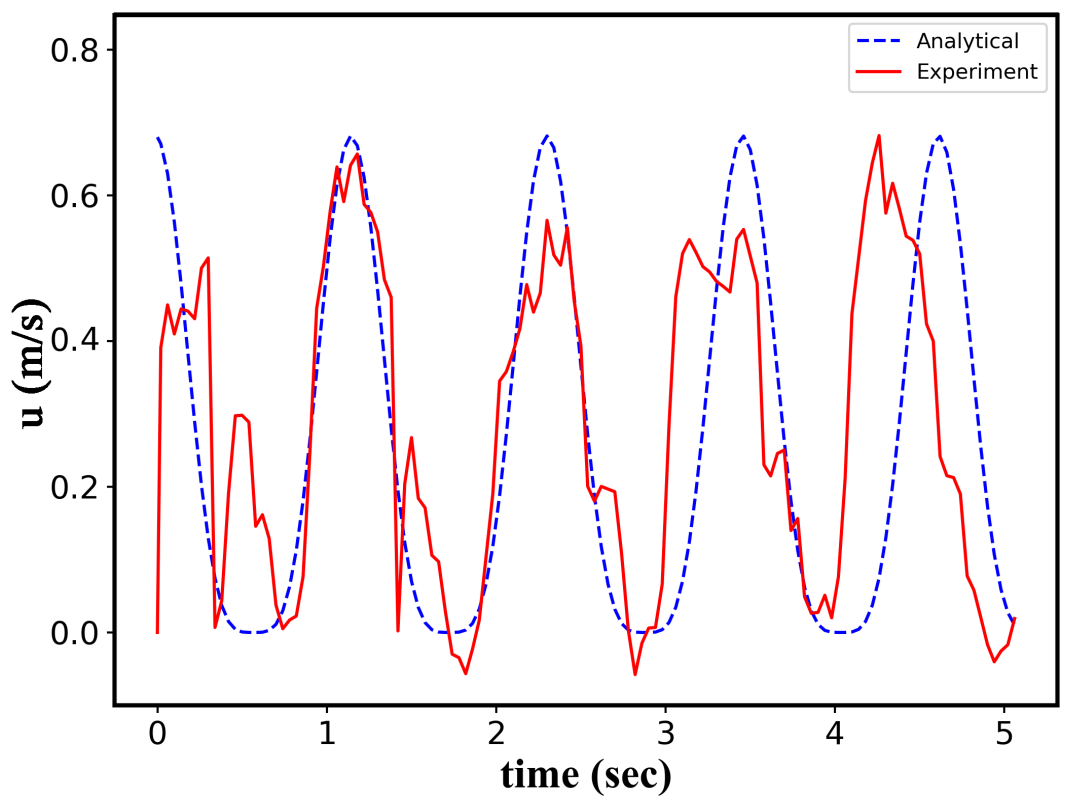

Figure 11. Cnoidal Wave solution for type of wave behind the bore, $\bar{h}=0.13 \mathrm{~m}$, $\Omega=5.347 \mathrm{rad} / \mathrm{s}, \omega=5.461 \mathrm{rad} / \mathrm{s}, \tau=0.03667$.

The captions of the previous figures show that the computed angular velocity $\omega$ in good accordance with the ones extracted experimentally only for relatively small rotation rate provided to the system, however in many cases the signal does not match perfectly, or only the first two waves match, the rest of the signal is shifted to the right, this can be ascribed to the increase in the rotation rate, and it could be a defect in the solution where we omitted the gravity forcing term to follow the direct integration, also it was noticed that the vectrino's place verti- 
cally may affect the signal, as mentioned in the previous section that one has to make it far from the bottom and from the free surface so that we can get smooth signal.

\section{Conclusion}

In this paper, some experimental results on the bore phenomenon were reported and compared with some analytical new models that were derived for this purpose. The experimental observations focused on the type of the free surface behind the bore and whether the classical norms can be used to classify it under precession conditions, the front of the bore was deviated towards the inner cylinder when increasing the rotation rate similar to other experiments carried out in [18] and [17] to study the rotation effect on hydraulic jumps, and this is due to the Coriolis effect. A new conjugate depth relationship was derived also, so that the rotation forces' effect was included, the new equation has time-space dependency, and it was compared with the experimental and the classical equation under nonrotation conditions. Other observations were on the velocity signals that were extracted from the ADV measurements, the signals all have Cnoidal form, thus a new BBM model was derived and integrated analytically, the agreement with the experiments is good for relatively medium rotation rate but it diverts when increasing the rotation speed.

\section{Acknowledgements}

The author is thankful for Professor. Szabó Gábor, Dr.Yiannis Hajimechaeal and Professor. Zsólt Kohári. The efforts of the laboratory staff of BME University are acknowledged for their help. The study of Hajar Alshoufi was covered by the Stipendium Hungaricum scholarship under contract number: SHE-14329-004/2017.

\section{Conflicts of Interest}

The author declares no conflicts of interest regarding the publication of this paper.

\section{References}

[1] V-Magana, M.R., Marchant, R.T., and Smyth, F.N. (2021) Numerical and Analytical Study of Undular Bores Governed by the Full Water Wave Equations and Bidirectional Whitham-Boussinesq Equations. Physics of Fluids, 33, Article ID: 067105. https://doi.org/10.1063/5.0050067

[2] Benjamin, B.T. and Lighthill, J.M. (1954) On Cnoidal Waves and Bores. Proceedings of the Royal Society A, 224, 448-460. https://doi.org/10.1098/rspa.1954.0172

[3] Keulegan, H.G. and Patterson, W.G. (1940) Mathematical Theory of Irrotational Translation Waves. Part of Journal of Research of the National Bureau of Standards, 24, 48-100. https://doi.org/10.6028/jres.024.027

[4] Binnie, M.A. and Orkney, C.J. (1955) Experiments on the Flow of Water from a Reservoir through an Open Horizontal Channel. Proceedings of the Royal Society $A$, 230, 237-246. https://doi.org/10.1098/rspa.1955.0126 
[5] Peregrine, H.D. and Svendsen, A.I. (1978) Spilling Breakers, Bores and Hydraulic Jumps. 16th International Conference on Coastal Engineering, Hamburg, $27 \mathrm{Au}-$ gust-3 September 1978, 30. https://doi.org/10.9753/icce.v16.30

[6] Bonneton, P., Van de Loock, J., Parisot, P.J., Bonneton, N., Sottolichio, A., Detandt, G., Castelle, B., Marieu, V. and Pochon, N. (2011) On the Occurrence of Tidal Bores-The Garonne River Case. Journal of Coastal Research, No. 64, 1462-1466.

[7] Treske, A. (1994) Undular Bores (Favre-Waves) in Open Channels-Experimental Studies. Journal of Hydraulic Research, 32, 355-370. https://doi.org/10.1080/00221689409498738

[8] Kikkert, A.G., O’Donoghue, T., Pokrajac, D. and Dodd, N. (2012) Experimental Study of Bore-Driven Swash Hydrodynamics on Impermeable Rough Slopes. Coastal Engineering, 60, 149-166. https://doi.org/10.1016/j.coastaleng.2011.09.006

[9] Mokhtar, A.Z., Yusuf, B., Mohammad, A.T. and Hamzah, B.S. (2019) Experimental Study of Tsunami Bore Induced Forces on Vertical Seawall. Pertanika Journal of Science and Technology, 27, 673-684.

[10] Trivellato, F. (2004) Experimental and Numerical Investigation of Bore Impact on a Wall. Transactions on the Built Environment, 71, 3-12.

[11] Chassagne, R., Filippini, G.A., Ricchiuto, M. and Bonneton, P. (2019) Dispersive and Dispersive-Like Bores in Channels with Sloping Banks. Journal of Fluid Mechanics, 870, 595-616. https://doi.org/10.1017/jfm.2019.287

[12] Lubin, P., Chanson, H. and Glockner, S. (2010) Large Eddy Simulation of Turbulence Generated by a Weak Breaking Tidal Bore. Environmental Fluid Mechanics, 10, 587-602. https://doi.org/10.1007/s10652-009-9165-0

[13] Chanson, Hurbert. and Docherty, J.N. (2012) Turbulent Velocity Measurements in Open Channel Bores. European Journal of Mechanics B/ Fluids, 32, 52-58. https://doi.org/10.1016/j.euromechflu.2011.10.001

[14] Peregrine, H.D. (1966) Calculations of the Development of an Undular Bore. Journal of Fluid Mechanicsh, 25, 321-330. https://doi.org/10.1017/S0022112066001678

[15] Alshoufi, E.H. (2021) On the Forced Oscillations in a Precessing Open Cylindrical Channel. AIP Advances, 11, Article ID: 045128. https://doi.org/10.1063/5.0046782

[16] Teles da Silva, F.A. and Peregrine, H.D. (1991) Nonsteady Computations of Undular and Breaking Bores. 22nd International Conference on Coastal Engineering, Delft, 1019-1032. https://doi.org/10.1061/9780872627765.079

[17] Pratt, J.L. (1987) Rotating Shocks in a Separated Laboratory Channel Flow. Journal of Physical Oceanography, 17, 483-491. https://doi.org/10.1175/1520-0485(1987)017\%3C0483:RSIASL\%3E2.0.CO;2

[18] Fedorov, V.A. and Melville, K.W. (1996) Hydraulic Jumps at Boundaries in Rotating Fluids. Journal of Fluid Mechanics, 324, 55-82. https://doi.org/10.1017/S0022112096007835 